\title{
Prácticas que obstaculizan los procesos de transposición didáctica en escuelas asentadas en contextos vulnerables: Desafíos para una transposición didáctica contextualizada
}

Beltrán Véliz, Juan; Navarro Aburto, Braulio; Peña Troncoso, Sebastián

Prácticas que obstaculizan los procesos de transposición didáctica en escuelas asentadas en contextos vulnerables: Desafíos para una transposición didáctica contextualizada

Revista Educación, vol. 42, núm. 2, 2018

Universidad de Costa Rica, Costa Rica

Disponible en: http://www.redalyc.org/articulo.oa?id=44055139023

DOI: https://doi.org/10.15517/revedu.v42i2.27571

Esta obra está bajo una Licencia Creative Commons Atribución-NoComercial-SinDerivar 3.0 Internacional. 


\title{
Prácticas que obstaculizan los procesos de transposición didáctica en escuelas
} asentadas en contextos vulnerables: Desafíos para una transposición didáctica contextualizada

\author{
Practices that Hinder the Processes of Didactic Transposition in Schools Sitters Vulnerable Contexts: Challenges \\ for a Didactic Transposition Contextualized
}

Juan Beltrán Véliz

Universidad Católica de Temuco, Chile

DOI: https://doi.org/10.15517/revedu.v42i2.27571

jbeltran@educa.uct.cl

Braulio Navarro Aburto

Universidad Autónoma de Chile, Chile

ademir@gmail.com

Sebastián Peña Troncoso

Universidad de Los Lagos, Chile

sebap988@hotmail.com

\author{
Redalyc: http://www.redalyc.org/articulo.oa?id=44055139023
}

Recepción: 25 Diciembre 2016

Aprobación: 20 Marzo 2018

\section{RESUMEN:}

Este artículo presenta el siguiente objetivo: Analizar las prácticas que obstaculizan los procesos de trasposición didáctica que desarrolla el profesorado en el aula en escuelas situadas en contextos vulnerables de la comuna de Curacautín de la Araucanía. Para ello, se planteó un diseño cualitativo descriptivo, con base en la teoría fundamentada y método comparativo constante. Los sujetos de estudio fueron 28 docentes de escuelas rurales. Las técnicas de recolección de información fueron la observación etnográfica y la entrevista semiestructurada. Los resultados del estudio indican que dentro de los prácticas que obstaculizan la transposición didáctica se evidencian modelos de planificación de enseñanza rígidos, la estrategia didáctica que se impone en el proceso de enseñanza en el aula es la clase expositiva centrada en el personal docente, con un enfoque vertical. Al mismo tiempo, se visualiza un débil manejo del dominio disciplinar y pedagógico del contenido. El proceso evaluativo, no se centra en la continuidad ni en la mejora del proceso de aprendizaje. Además, se visualiza ausencia de liderazgo pedagógico. Por lo tanto, se concluye que los procesos de transposición didáctica tienen una orientación conductista, y descontextualizada. En tal sentido, se espera de que los grupos docentes rompan con la forma tradicional de enseñanza, para facilitar los procesos de transposición didáctica con base en estrategias y recursos didácticos contextualizados, a fin de mejorar los procesos de enseñanza-aprendizaje y, por tanto, provocar transformaciones sociales en contextos de vulnerables.

Palabras ClaVE: Didáctica, transposición didáctica, estrategias didácticas, vulnerabilidad.

\section{AbStract:}

The objective of this article is analyzing the practices hindering the processes of didactic transposition that teachers develop in the classroom in schools located in vulnerable contexts in the Curacautín de la Araucania community. For this purpose, a descriptive qualitative design based on grounded theory and a constant comparativemethod were used. The study subjects were 28 teachers from rural schools. The data collection techniques used were ethnographic observation and semi-structured interviews. The study results indicate that among the practices hindering didactic transposition, rigid planning teaching models are evident. The teaching strategy that is imposed in the classroom is the traditional expository teacher-centered lesson. At the same time, poor classroom management and pedagogical content are visualized. Assessment is neither ongoing not focused on improving the learning process. In addition, a lack of pedagogical leadership is present. Therefore, it is concluded that the processes of didactic transposition have a behavioral decontextualized orientation. In this regard, teachers are expected to break with the traditional way of teaching to facilitate the processes of didactic transposition based on contextualized strategies and teaching resources to improve the teachinglearning process, and thus leading to social transformations in vulnerable contexts.

KEYWORDS: Didactic, didactic transposition, teaching strategies, vulnerability. 


\section{INTRODUCCIÓN}

"En la actualidad, resulta un reto para el profesorado el desarrollo de procesos didácticos que orienten el aprendizaje de los alumnos de tal manera que puedan responder a las demandas de la sociedad contemporánea" (Mansilla y Beltrán, 2013, p.26).

La falta de equidad en la educación chilena es un problema, dado que se expresa en la falta de procesos, estructura y resultados que traduzcan buenas prácticas y, por ende, favorezcan el aprendizaje de las instituciones educativas y, principalmente, del estudiantado (Becerra, Mansilla, Saavedra y Tapia, 2012). En conexión con lo expuesto, la calidad del personal docente y de su enseñanza es el factor más importante para explicar los resultados de estudiantes (OCDE, 2004). "Existe un consenso emergente acerca de que el profesorado influye de manera significativa en el aprendizaje de los alumnos y en la eficacia de la escuela" (Cochran-Smith y Fries, 2005, p.40). Tales argumentos cobran fuerza, dado que la educación actual está caracterizada por la diversidad de contextos, lo cual es una problemática permanente en el desarrollo de prácticas pedagógicas de docentes que se desempeñan en contextos vulnerables. Desde esta perspectiva, la intervención del profesorado en el acto didáctico es trascendental, pues la enseñanza de saberes queda sujeta a las capacidades y competencias pedagógicas de docentes.

En este contexto, Aylwin, Muñoz, Flanagan y Ermter (2005) expresan que escasas escuelas se hacen la pregunta sobre cómo enseñar en contextos vulnerables en donde se desempeñan. Del mismo modo, se visualizan dificultades y escasez en el manejo de estrategias y herramientas metodológicas y didácticas: profesorado que no sabe qué y cómo enseñar. En concordancia, en un estudio realizado por Aroca (2015), se muestra que los grupos docentes no poseen todas las herramientas necesarias para enfrentar el trabajo con grupos de vulnerabilidad, lo que hace que no se involucren en forma activa a los diferentes ambientes de aprendizaje.

De acuerdo con lo anterior, avanzar hacia la mejora de la calidad de la educación ha sido el foco de las reformas educativas en diversos países, y en ello se reconoce que a nivel de instituciones educativas el factor "profesorado" como una de las variables que mayor peso tienen sobre los resultados de aprendizaje estudiantil (Becerra et al. 2012). En consecuencia, "los desafíos para enseñar en contextos de vulnerabilidad implican un alto nivel de especialización de los docentes y coherencia de acciones al interior de las escuelas" (UNESCO, 2010, p. 8). Es decir, enseñar en contextos vulnerables exige a docentes una formación profesionalizante y, por tanto, un manejo adecuado del conocimiento pedagógico. Para ello, es necesario realizar ciertas transformaciones en sus prácticas pedagógicas. En tal sentido, se debe producir una transformación de saberes sabios, lo cual conlleva a cambios paradigmáticos, donde debe ocurrir una verdadera revolución científica y provocar una crisis a fin de tensionar al paradigma dominante, y dar paso al surgimiento de núcleos teóricos emergentes, (Khun, 1962). Por tanto, se debe provocar un cambio de paradigma en la forma de enseñar en diversos contextos. Desde esta perspectiva, se produce una yuxtaposición entre la tradición pedagógica y la innovación curricular.

En este contexto, surge la propuesta de transposición didáctica, donde el profesorado construye un conocimiento acerca de la disciplina, busca los sentidos, formas y medios para enseñar aquel contenido que pretende construir con sus estudiantes y posee diversos conocimientos que están presentes en todo momento en su quehacer profesional (Chevallard, 1991). En esta lógica, este autor señala que se "puede considerar la existencia de una transposición didáctica, como proceso conjunto, como situaciones de creaciones didácticas de objetos (de saber y de enseñanza a la vez) que son 'necesarias' por las exigencias del funcionamiento didáctico” (p. 47). En tal sentido, el profesorado debe apropiarse de competencias que le permitan trasladar el saber disciplinar hacia un saber pedagógico, de tal forma que sus estudiantes adquieran ese conocimiento, pues el rol docente en el aula y en el caso de contextos educativos vulnerables es determinante en la calidad del proceso de enseñanza y aprendizaje. 
En este campo, cobra sentido la propuesta del conocimiento pedagógico del contenido cuyo origen está en el denominado conocimiento didáctico del contenido (CDC) propuesto por Lee Shulman. Al respecto, Bolivar (2005) señala que "en el modelo de Shulman, además del conocimiento de la materia y del conocimiento general pedagógico, los profesores deben desarrollar un conocimiento específico: cómo enseñar su materia específica" (p. 7), por lo que se requiere un CDC, propio del buen hacer docente. Desde esta perspectiva, el CDC facilita la comprensión del profesorado, dado que cuando "conoce una materia" se convierte poco a poco en "maestro maestra [CB1] de la materia" mediante la utilización de una serie de recursos y estrategias didácticas (Clermont, Borko y Krajcik, 1994; Marcelo, 2001; Mulholland y Wallace, 2005). Al respecto, Shulman (1986) sostiene que para los tópicos más regularmente enseñados en un área de contenido, se debe incluir las formas de representación y formulación del tema que lo hace comprensible a otras personas. Además, este autor señala que "[CB2] el profesorado necesita el conocimiento de las estrategias que más probablemente funcionen en la reorganización del entendimiento de sus estudiantes" (Shulman, 1986, p. 9). En esta lógica, se le exige al personal docente disponer de un dominio del conocimiento científico y de cómo hacerlo apto para la enseñanza, mediante la utilización de estrategias y recursos didácticos adecuados, para un contexto determinado.

En este plano, se hace necesario que el profesorado incorpore en sus prácticas de enseñanza no solamente el dominio de contenido disciplinario puro, sino que además, se exigen competencias asociadas a la apropiación del conocimiento didáctico para vehiculizar el saber disciplinario hacia el saber pedagógico, con el objeto de cumplir con el acto educativo (Pellón, Mansilla, y San Martín, 2009). Por lo tanto, es esperable que el profesorado tenga un dominio acabado de la transposición didáctica de contenido. Desde esta mirada, la transposición didáctica cobra relevancia, puesto que se requiere que cada docente posea un dominio disciplinar y pedagógico adecuado sobre la base de estrategias de enseñanza y aprendizaje, recursos didácticos e instrumentos de evaluación, para lo cual se deben tener en cuenta las características del contexto educativo.

En razón de las evidencias empíricas y discusión teórica surge el siguiente objetivo: Analizar las prácticas que obstaculizan los procesos de trasposición didáctica que desarrolla el profesorado en el aula en escuelas situadas en contextos vulnerables de la comuna de Curacautín de la Araucanía.

\section{DidÁCTICA}

En el siglo XVII emerge el teórico considerado como el padre de la didáctica: el obispo protestante Juan Amos Comenio (1592-1670), quien fue una de las personas excepcionales en la pedagogía (Böhm y Schifelbein, 2010). En su obra, Didáctica magna, (Gran didáctica), señala que la didáctica es entendida como un método universal de enseñanza (Grizales-Franco y González-Aguadelo, 2009). Pero es en la década de los setenta que esta cobra especial relevancia, al respecto Verret (1975, p. 139) la define como "la transmisión de aquellos que saben a aquellos que no saben. De aquellos que han aprendido a aquellos que aprenden”. Tal afirmación cobra importancia en la transmisión de un determinado saber desde alguien que sabe a quien no tiene noción de dicho saber. En este sentido, Morgado (2007) señala que la didáctica es el arte de enseñar o dirección técnica del aprendizaje. Es parte de la pedagogía que describe, explica y fundamenta los métodos más adecuados y eficaces para conducir al estudiantado a la progresiva adquisición de hábitos, técnicas para una formación integral. La didáctica es la acción que el personal docente ejerce sobre la dirección del educando, para que este llegue a alcanzar los objetivos de la educación. En este sentido, la didáctica permite buscar unas técnicas y estrategias que facilitan enseñar y lograr los objetivos de una forma más efectiva. Al respecto, De la Torre (2005) dice que la didáctica se define como la técnica que se emplea para manejar, de la manera más eficiente y sistemática, el proceso de enseñanza-aprendizaje. En consecuencia, estos autores solo se refieren a la didáctica desde una perspectiva metodológica para vehiculizar los saberes y lograr los objetivos propuestos desde un perspectiva eficiente. Complementando lo anterior, Marhuenda (2000) sostiene que la didáctica cobra 
sentido al ocuparse tanto de la selección y distribución del contenido, lo estrictamente curricular, como la transmisión de este y la forma de cómo enseñar.

En relación con lo anterior, la didáctica no debe concebirse únicamente como un variado conjunto de estrategias docentes amplias y coherentes con la finalidad de que el profesorado pueda aplicarlas en el contexto del aula (Moreno, 2011). La reducción de la didáctica a la vertiente metodológica, en efecto, deriva su identidad a un ángulo prescriptivo, de juicios de valor sobre lo que se deba hacer, desdeñando la dimensión de teorizar los fenómenos de la enseñanza. En este sentido, en un estudio realizado, Mansilla y Beltrán (2013) constatan que la didáctica se reduce al plano metodológico, pues no aparece el concepto de conocimiento didáctico como un todo. Esto implica un reduccionismo de la didáctica, pues se restringe su accionar, llevándola a un plano técnico, carente de un proceso educativo integral. Por otro lado, según Zambrano (2006), la didáctica es una disciplina científica cuyo objeto es el estudio de la génesis, circulación y apropiación del saber y sus condiciones de enseñanza y aprendizajes. Este objeto se vuelve específico y tiene sus raíces en la especificidad de los saberes escolares y disciplinares. El saber escolar se entrelaza con el saber disciplinar y exige un referente disciplinar específico. Por su parte, Camilloni, Cols, Besabe y Feeney (2007) plantean que la didáctica es una disciplina teórica que se ocupa de estudiar la acción pedagógica, es decir, las prácticas de la enseñanza, y que tiene como misión describirlas, explicarla, fundamentar y enunciar normas para la mejor resolución de los problemas que estas prácticas plantean a docentes. Estos estudios abordan la didáctica como una teoría y disciplina, donde las prácticas de enseñanza tienen un papel activo, argumentativo, integrador, reflexivo, creativo en la resolución de problemas desde las prácticas docentes.

En este campo, Marhuenda (2000) sostiene que la didáctica cobra sentido al ocuparse tanto de la selección y distribución del contenido, lo estrictamente curricular, como la transmisión de este y la forma de cómo enseñar. Desde esta perspectiva, la didáctica debe ocuparse de la selección del contenido, y de las formas de enseñanza apropiadas al contexto estudiantil. En esta línea, Medina (citado en Mansilla y Beltrán, 2013) señala que la didáctica es la disciplina rigurosa de estudio y fundamentación de la actividad de enseñanza en cuanto propicia el aprendizaje formativo de estudiantes en los más diversos contextos. En tal sentido, la didáctica favorece la mejora de los procesos de transposición didáctica llevada a cabo por el profesorado, debido a que la enseñanza debe centrase en las características y necesidades educativas estudiantiles, para contribuir a la generación de aprendizajes significativos en alumnado situado en diversos contextos.

En correspondencia, la didáctica es más que unas técnicas y metodologías para desarrollar el acto didáctico, pues Gallego y Salvador (2012, p. 118) sostienen que “toda programación didáctica incluye una serie de elementos esenciales: (a) Los objetivos, (b) Los contenidos, (c) La metodología (estrategias didácticas), (d) Los recursos didácticos, y (e) La evaluación”. En esta lógica, Moreno, Rodríguez, Mera y Beltrán (2007) señalan que al hablar de didáctica se tiene que involucrar sus elementos fundamentales como son: (i) estudiante, (ii) personal educador, (iii) los objetivos, (iv) la metodología, (v) los recursos y (vi) la evaluación. Elementos que necesariamente se ajustan al momento histórico, cultural y social. Por lo tanto, la didáctica es una disciplina que incorpora una serie de componentes que facilitan, al profesorado, llevar a cabo la transposición de saberes contextualizados, de forma eficiente para lograr los objetivos propuestos. Asimismo, permitiría dar respuesta a las necesidades de una educación sustentada en contextos diversos, desde una perspectiva social y cultural.

\section{TransPosición DIDÁCTICA}

Desde el campo de la didáctica, emerge la propuesta de transposición didáctica. Esta idea fue formulada originalmente por el sociólogo Michel Verret en 1975. Este autor, señala que no se puede enseñar un objeto sin transformación: "toda práctica de enseñanza de un objeto presupone, en efecto; la transformación previa de su objeto en objeto de enseñanza” (Verret, 1975, p. 140). Esto implica seleccionar el saber, además este debe experimentar una transformación para enseñarlo adecuadamente. El matemático, Yves Chevallard, retoma 
el postulado de transposición didáctica de Verret desde una aproximación antropológica de los saberes. Al respecto, Chevallard (1991) la define como:

Un contenido de saber que ha sido designado como saber a enseñar, sufre a partir de entonces un conjunto de transformaciones adaptativas que van a hacerlo apto para ocupar un lugar entre los objetos de enseñanza”. El "trabajo" que transforma de un objeto de saber a enseñar en un objeto de enseñanza, es denominado la transposición didáctica. (p. 45)

Respecto a tal afirmación, la transposición didáctica se produce cuando el saber sufre una serie de transformaciones para ser enseñable. En esta línea, "la transposición didáctica tiene lugar cuando pasan al saber enseñado elementos del saber" (Chevallard, 1991, p. 25). Es decir, el saber científico sufre una serie de transformaciones apropiadas para ser enseñable al estudiantado. En concordancia, Tardy (1995, p. 53) lo describe como "el recorrido que va del saber académico (saber que se elabora en los lugares consagrados a la investigación) al saber enseñado (el saber propuesto a los alumnos)”. Entonces, la transposición didáctica experimenta adaptaciones sistemáticas, a través de las cuales el conocimiento disciplinar se transforma en conocimiento a enseñar y este en conocimiento apto para ser enseñado. En este sentido, la responsabilidad del profesorado es transformar el saber sabio (o sea el saber científico o artístico) en un saber enseñado con el fin de que sus estudiantes puedan comprender ese lenguaje y puedan apropiarse de este conocimiento (Grizales-Franco y González-Aguadelo, 2009). Por tanto, el proceso de transposición didáctica implica un proceso de transformación de saberes científicos en saberes enseñados, donde se deben tener en cuenta las características y el contexto estudiantil, con la finalidad de dar sentido a los conocimientos a enseñar con base en estrategias de enseñanza y aprendizajes, actividades, recursos didácticos, e instrumentos de evaluación, para que el estudiantado logre apropiarse de esos saberes.

En este escenario, emerge un postulado similar al de transposición didáctica: el conocimiento didáctico del contenido (CDC). Este adquiere particular interés porque identifica los cuerpos de conocimientos distintivos para la enseñanza. "[CB3] Representa la mezcla entre materia y didáctica por la que se llega a una comprensión de cómo determinados temas y problemas se organizan, se representan y se adaptan a los diversos intereses y capacidades del alumnado, y se exponen para su enseñanza" (Shulman, 2005, p.11[CB4]). En este campo, el CDC implica un conjunto de saberes que permite al profesorado trasladar, a la enseñanza, el contenido de un determinado tópico; esto es, hacer la transposición didáctica del conocimiento especializado de un tema al conocimiento escolar objeto de enseñanza y aprendizaje (Acevedo, 2009). Entonces, el conocimiento didáctico se entrelaza entre el contenido y pedagogía para organizar y llevar a cabo la enseñanza de forma adecuada mediante el uso de estrategias, técnicas y recursos didácticos que toman en cuenta las habilidades, capacidades, destrezas y las características del estudiantado.

Respecto a lo expuesto, en el contexto de vulnerabilidad educativa toma relevancia la transposición didáctica contextualizada y el CDC. En tal sentido, el modelo integrador del CDC desarrollado por GessNewsome (1999) adquiere pertinencia, pues es el resultado de la intersección entre la didáctica, el contenido y el contexto (Fig. 1). Este modelo considera tipos de conocimiento que se constituyen en conceptos claves para yuxtaponer los saberes disciplinarios, didácticos y contextuales; complejiza la actuación docente, donde hay un desafío a la superación de una enseñanza magistocéntrica, de tal forma que esta se oriente a una lógica paidocéntrica (Pellón et al. 2009), es decir, transitar a una enseñanza focalizada en el personal docente a una enseñanza centrada en el estudiantado tomando en cuenta el conocimiento del tema, conocimiento didáctico del contenido y el contexto, a fin que este le de sentido a lo que aprende. 


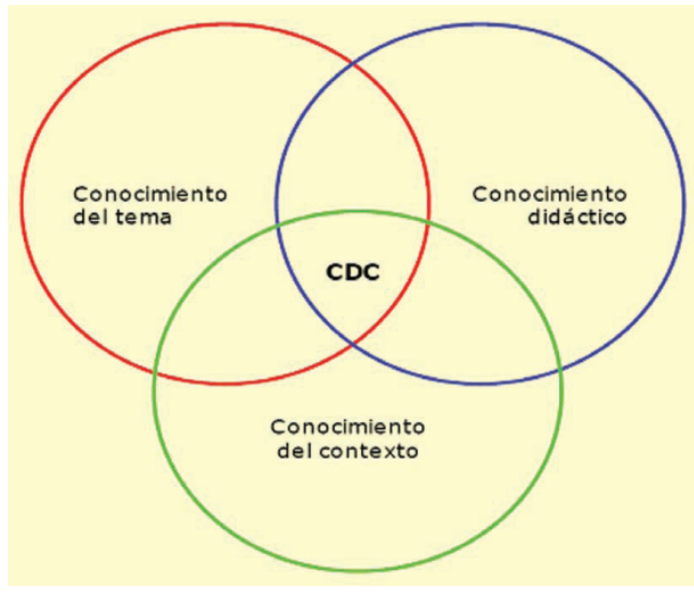

FIGURA 1

Modelo integrador del CDC

Gess-Newsome (1999)

Las interacciones que se aprecian en la figura anterior permiten la "transformación del contenido para su enseñanza;" es decir, la transposición didáctica del contenido (Chevallard, 1991). Por tanto, en la propuesta del CDC de Shulman, además del conocimiento de la materia y del conocimiento general pedagógico, el profesorado debe desarrollar un conocimiento específico: cómo enseñar su materia específica, por lo que se requiere un CDC, propio del buen hacer docente (Bolivar, 2005). En este sentido, el compromiso del profesorado es transformar el saber científico en un saber enseñado, para lo cual se le exige al personal docente disponer de un dominio del conocimiento científico y de cómo hacerlo apto para la enseñanza, mediante la utilización de medios, estrategias y recursos didácticos adecuados a las características, estilos de aprendizaje y necesidades educativas de estudiantes, tomando en cuenta su contexto.

\section{VULNERABILIDAD EN EL CONTEXTO EDUCATIVO}

En el contexto de vulnerabilidad, en el cual el personal docente desarrolla sus prácticas pedagógicas, es preciso abordar el concepto la vulnerabilidad educativa. Para aproximarse a tal acepción, primero es necesario abordar la acepción de vulnerabilidad. El MIDEPLAN (2002) lo define como el proceso multidimensional que confluye en el riesgo o probabilidad del individuo, hogar o comunidad, de ser herido, lesionado o dañado ante cambios o permanencia de situaciones internas o externas. En esta lógica, la vulnerabilidad alude a "las características de una persona o grupo y su situación, que influencian su capacidad de anticipar, lidiar, resistir y recuperarse del impacto de una amenaza" (Wisner, Blaikie, Cannon y Davis, 2004, p. 11). Por su parte, León (2011) señala que la vulnerabilidad es la situación de dependencia en que pueden estar personas o grupos sociales, que pone en riesgo la autodeterminación y la libre elección en sus ideales de vida y en su desarrollo. Entonces, la vulnerabilidad obstaculiza y pone en riesgo el desarrollo del individuo en diversos aspectos. Desde esta perspectiva, surge la noción de "vulnerabilidad educativa", definida por la Dirección General de Cultura y Educación (2009) como: el conjunto de condiciones (materiales y simbólicas, de orden objetivo y subjetivo) que debilitan el vínculo de escolarización del alumnado. Asimismo, exige dar cuenta de las interacciones entre estas dimensiones, con particular atención a aquellos factores propiamente escolares.

El concepto de "vulnerabilidad educativa" permite analizar la complejidad de las problemáticas relacionadas con las trayectorias escolares y el vínculo de escolarización de niños, niñas y jóvenes. La perspectiva de la "vulnerabilidad educativa" implica atender posibles interrupciones y desfases en las trayectorias escolares del alumnado desde las propias instituciones escolares (Dirección General de Cultura y Educación, 2010). Asimismo, la vulnerabilidad educativa es interpretada como una derivación negativa 
originada por las características socioculturales del hogar, que actúan sobre el rendimiento y desempeño educativo (Giberti, Garaventa y Lamberti, 2005). En concordancia, está presente como algún tipo de desvalimiento que es interiorizado y demostrado como carencia en el actuar en el aula o que opera orientando negativamente las trayectorias escolares (Sabuda, 2009). Respecto a lo anterior, cabe señalar que la vulnerabilidad educativa dificultaría el desarrollo del proceso de enseñanza-aprendizaje del estudiantado $\mathrm{y}$, por tanto su formación y educación desde un plano de desigualdad, equidad y, de igual forma, en el aspecto social y cultural durante el desarrollo de su vida.

\section{Metodología}

Considerando las características y el propósito del estudio, se sustenta en el enfoque metodológico cualitativo, pues propone una forma de construcción del conocimiento, basándose en la subjetividad e intersubjetividad, en el contexto en que ocurren los fenómenos y se sirve de la descripción e interpretación para la comprensión de fenómenos socioculturales (Sandín, 2003; Valles, 2007). En correspondencia, se usó la teoría fundamentada (Strauss y Corbin, 2002), para abordar el proceso de análisis mediante la construcción de categorías conceptuales, a través de la utilización del método comparativo constante (en adelante MCC) (Mansilla y Beltrán, 2013). Este procedimiento se emplea para interpretar textos consistentes en "cuidar de comparar los códigos una y otra vez con los códigos de las clasificaciones obtenidas” (Flick, 2007, p. 248). Además, se empleó el estudio de caso, que hace referencia al "foco de atención que se dirige a un grupo de conductas o personas, con el propósito de comprender su ciclo vital, sea esta una unidad individualizada, un grupo, una institución social o una comunidad" (Cerda, 1998, p. 85).

El caso está constituido por 28 docentes de segundo ciclo de educación primaria de tres escuelas rurales situadas en contextos vulnerables de la comuna Curacautín de la Araucanía. La selección de este grupo participante fue intencionada, al respecto Gurdián-Fernández (2007, p. 247) señala que "la selección debe ser apropiada a las y los sujetos actuantes. Es decir, seleccionar a quienes tengan un mejor conocimiento del fenómeno por investigar. Esto garantiza una saturación efectiva y eficiente de las categorías con información de óptima calidad". En este contexto, se presentan en la Tabla 1, los criterios de inclusión de participantes.

TABLA 1

Criterios de inclusión de participantes

\begin{tabular}{ll}
\hline Criterios & Descripción \\
\hline Género & Femenino y masculino. \\
Rol o profesión que desempeña & Docente de educación primaria. \\
Ciclo & Segundo ciclo: Quinto a octavo de educación primaria. \\
Experiencia profesional & Mínimo 2 años en aula. \\
Contexto & Vulnerabilidad enmarcada en la ruralidad. \\
\hline
\end{tabular}

Nota: Elaboración propia (2017).

Para recoger la información se emplearon las siguientes técnicas: la entrevista semi-estructurada y la observación etnográfica. Respecto de la primera, Ruiz (2003) señala que la entrevista semi-estructurada permite a quien investiga dar la oportunidad de que el sujeto entrevistado pueda expresarse en sus propios términos y con la suficiente profundidad para captar toda la riqueza de su significado. En cuanto a la observación etnográfica, Flick (2007) plantea que nace del deseo por parte de quien investiga de ir más allá de la palabra hablada y del informe sobre las acciones, a favor de analizar estas a medida que se producen de modo natural. Quien hace etnografía debe pasar el tiempo suficiente en el escenario, de manera que pueda ver lo que sucede en repetidas ocasiones. 
La salida del trabajo de campo se realizó cuando se produjo la "saturación teórica”. En esta etapa los datos se tornaron repetitivos, en conexión Flick define el criterio de "saturación teórica" de la siguiente manera: "el muestreo e integración de material nuevo se acaba cuando la 'saturación teórica' de una categoría o grupo de casos se ha alcanzado, es decir, cuando no emerge ya nada nuevo” (Flick, 2007, p. 79).

\section{ANÁLISIS}

El análisis de la información obtenida de las entrevistas semi-estructuradas y las observaciones etnográficas se realizó mediante la utilización del programa informático Atlas ti 7.0., el cual, además de facilitar el análisis de los documentos primarios, permite organizarlos, agruparlos y elaborar anotaciones sobre estos (Mayorga, Enrique y Rodríguez, 2014). La técnica utilizada fue el análisis del contenido, basado en la teoría fundamentada para interpretar los datos que emergen de las entrevistas y de las observaciones. Los datos fueron reducidos en el proceso de codificación abierta (Mansilla y Beltrán, 2013). Al respecto, Strauss, \& Corbin (2002, p. 78) señalan "que para descubrir y desarrollar los conceptos debemos abrir el texto y exponer los pensamientos, ideas y significados contenidos en él”. En este sentido, Bisquerra (2004) menciona que debe sumergirse literalmente en el documento o situación para identificar los temas o las dimensiones más relevantes. Durante este proceso de codificación abierta, se levantaron las categorías desde el procedimiento inductivo (Mansilla y Beltrán, 2013). Las categorías son numeradas consecutivamente, siguiendo el orden de la recogida de datos, estos fueron examinados y comparados en términos de sus similitudes y diferencias, donde se hace una descripción de las categorías en busca de los referentes del marco teórico y los temas que emergieron de los propios sujetos con el MCC (Mansilla y Beltrán, 2013). En este contexto, se definen las categorías que emergen del análisis en la Tabla 2.

TABLA 2

Descripción de las categorías que emergen del análisis

\begin{tabular}{|c|c|}
\hline Criterios & Descripción \\
\hline \multirow{2}{*}{$\begin{array}{l}\text { Diseño de la planificación } \\
\text { de enseñanza }\end{array}$} & $\begin{array}{l}\text { Alude a la organización de la enseñanza que al personal docente le correspondería } \\
\text { realizar en conjunto con sus pares. }\end{array}$ \\
\hline & $\begin{array}{l}\text { Asimismo, debería estar centrada en estrategias didácticas actualizadas, en las } \\
\text { características educativas y en el contexto estudiantil. }\end{array}$ \\
\hline Estrategias didácticas & $\begin{array}{l}\text { Hace referencia a procedimientos, estructuras de actividades que el profesorado } \\
\text { debería emplear con el propósito de lograr unos contenidos y objetivos de } \\
\text { aprendizaje. }\end{array}$ \\
\hline $\begin{array}{l}\text { Dominio del contenido } \\
\text { disciplinario }\end{array}$ & $\begin{array}{l}\text { Corresponde al manejo que tiene el personal docente de los contenidos } \\
\text { disciplinarios, es decir, el dominio del saber que el profesorado debería poseer } \\
\text { en el desarrollo de sus prácticas pedagógicas en determinadas asignaturas. }\end{array}$ \\
\hline Concepción de evaluación & Alude al significado que para docentes tiene la evaluación. \\
\hline Prácticas evaluativas & $\begin{array}{l}\text { Se refiere a las prácticas evaluativas y tipos de evaluación que lleva cabo el } \\
\text { profesorado, durante el desarrollo de los procesos de transposición didáctica. }\end{array}$ \\
\hline \multirow[b]{2}{*}{ Liderazgo pedagógico } & $\begin{array}{l}\text { Alude a la capacidad de trabajar en equipo que debería realizar el profesorado, } \\
\text { haciendo uso de un lenguaje habitual, con el fin de lograr objetivos en común. }\end{array}$ \\
\hline & $\begin{array}{l}\text { Además, este tipo de liderazgo debería centrase en el alumnado, es decir, en las } \\
\text { características, necesidades educativas, trabajo en equipo entre estudiantes y } \\
\text { en el contexto educativo, a fin de mejorar la calidad de los aprendizajes. }\end{array}$ \\
\hline
\end{tabular}

Nota: Elaboración propia (2017).

Luego, en una segunda fase, mediante una matriz de comparación, se identificaron las relaciones entre categorías en el proceso de codificación axial. Este proceso puede ser entendido, según Strauss y Corbin (2002), como el acto en el cual las categorías se interrelacionan y se vinculan. Para (Flick, 2007), este 
proceso depura y diferencia las categorías derivadas de la codificación abierta. Las categorías axiales se enriquecen por su ajuste con el mayor número de pasajes posibles, se relacionan las categorías y sus subcategorías. Consecutivamente, se dio paso a la generación de una red conceptual que resume los conceptos fundamentales y sus relaciones que igualmente son descritas. Luego, se realizó un muestreo teórico empírico, asimismo se utilizó la triangulación como criterio de rigor. Al respecto, Denzin (1970) define la triangulación en investigación como: la combinación de dos o más teorías, fuentes de datos, métodos de investigación, en el estudio de un fenómeno singular. Para ello, se empleó la triangulación de datos y metodológica. Respecto a la primera, Denzin (1989) se refiere a la utilización de diferentes fuentes de datos, y en cuanto a la triangulación metodológica, alude a la combinación de técnicas, ejemplo: combinar la observación etnográfica con una entrevista semiestructurada. Posteriormente, se realizó el análisis y discusión de resultados, y finalmente se presentan las conclusiones.

\section{ANÁLISIS Y DISCUSIÓN DE RESULTADOS}

A partir la codificación abierta y axial surgieron seis categorías: 1) Diseño de la planificación de enseñanza, 2) Estrategias didácticas, 3) Dominio del contenido disciplinario, 4) Concepción de evaluación, 5) Prácticas evaluativas, y 6) Liderazgo pedagógico.

\section{Categoría 1. Diseño de la planificación de enseñanza}

En esta categoría se visualizan planificaciones de enseñanza centradas en el individualismo, con un diseño rígido y predeterminado: “...cuando tú planificas sobre todo en las clases teóricas donde hay que pasar ciertos puntos (...) a lo más puedes hablar más, puedes hablar otras cosas también, pero de esos puntos no te puedes salir..." (E: P4). Además, no se observa comisiones de trabajo en equipo para elaborar planificaciones, que permitan intercambiar ideas y experiencias educativas entre docentes. Lo señalado, se refleja en la siguiente textualidad: "Nosotros generalmente planificamos individualmente, esto porque no existen la conformación de equipos de trabajo ni el tiempo para llevarlo a cabo" (E: P4).

La elaboración de las planificaciones apela a criterios intuitivos, experienciales y a las creencias. Igualmente es posible apreciar el desconocimiento que tiene el profesorado del contexto, de las habilidades, capacidades, ritmos y estilos de aprendizajes de sus estudiantes a la hora de planificar. Tales evidencias dan cuenta de la rigidez con que algún profesorado realiza sus planificaciones de manera que no responde a un diseño global e integrado, donde se considere el "CDC como resultado de la intersección entre la didáctica, el contenido y el contexto" (Gess-Newsome, 1999). En este campo, además, se evidencia falta de reflexión pedagógica entre docentes en el diseño de la planificación. En contraste, Schön (2011) plantea que la práctica profesional reflexiva permite al personal docente la construcción de conocimientos a través de la solución de problemas que se encuentran en la práctica; esto conlleva a la construcción de un tipo de conocimiento desde las acciones para tomar decisiones mediante la utilización de estrategias y metodologías para innovar. En tal sentido, el diseño de la planificación debería estar orientada al estudiantado que está en situación de vulnerabilidad, para lo cual se deben trabajar los contenidos a enseñar desde una postura colaborativa, y reflexiva entre docentes, mediante el intercambio de estrategias didácticas, experiencias educativas, ideas, recursos didácticos y tipos de evaluación para atender los diversos estilos y ritmos de aprendizaje. Además, como afirma Castro, Domínguez, Manzano, Zavala, Sánchez y Guzmán (2015) se debe trabajar con aquel estudiantado vulnerable en diferentes ambientes de aprendizaje. En este sentido, el diseño de la planificación no debe dejar espacios para la improvisación y las creencias. En concordancia, uno de los factores que más influye en los buenos resultados que obtienen escuelas en condiciones vulnerables es el desarrollo de una planificación que no deja espacios para la improvisación (Aylwin, Muñoz, Flanagan y Ermter, 2005). En esta lógica, el diseño de la 
planificación debería experimentar un cambio, centrada en una pedagogía flexible, inclusiva y con un sentido de pertinacia.

Además, se observa que el proceso de enseñanza y aprendizaje, se dificulta, debido a la ausencia de condiciones de organización óptimas de parte de personal directivo para llevar a cabo un trabajo en equipo entre docentes. Al respecto, el trabajo del equipo directivo debería vincularse directamente con el mejoramiento de las condiciones organizacionales de los actores y de los resultados educativos (Fullan, 2003; Murillo, 2005; Raczynski y Muñoz, 2005). Esto, con el fin de mejorar el trabajo colectivo entre profesorado $y$, por tanto, favorecer los procesos de enseñanza-aprendizaje de estudiantes en condiciones vulnerables.

Respecto de esta categoría, se evidencia una enseñanza basada en el estilo tradicional, se visualiza un uso excesivo de las clases expositivas. Las estrategias didácticas están centradas en el personal docente, promueven actitudes poco reflexivas en su quehacer. Del mismo modo, no existen espacios para la reflexión y la retroalimentación de los aprendizajes. Lo anterior da cuenta de la rigidez con que una parte del profesorado realiza sus clases, en concordancia, un entrevistado en este punto declara: “...uno tiene que mantener el orden en la sala, uno es el que hace la clase, uno es el que dicta las materias" (E: P12). En tal sentido, el rol del profesorado y de estudiantes se encuentra definido de manera rígida y asimétrica, donde el grupo profesional tiene una figura de autoridad, es el poseedor del conocimiento y de la verdad absoluta y, el estudiantado es considerado un actor pasivo. En conexión con la evidencia empírica anterior, se observan recuadros proyectados. Esto se visualiza en la siguiente evidencia empírica: "La mayoría de las veces uso Power Point, que es una herramienta nueva y bastante fácil de manejar y de hacer una clase" (E: P15). Este método ayuda más bien a cada docente a dictar o leer la clase proyectada, pero no contribuye al entendimiento de los conceptos por parte de sus estudiantes, pues está centrado en el docente y promueven espacios poco reflexivos y críticos en beneficio de la construcción de los aprendizajes. En este contexto, De Miguel-Díaz (2005) señala que para propiciar un cambio de paradigma de la enseñanza-aprendizaje, es fundamental que el profesorado facilite las herramientas necesarias para que el estudiantado lleve a cabo la búsqueda personal hacia la mejora de su desempeño estudiantil, cuestión que facilitaría el desarrollo de su autonomía como sujeto cognoscente. En este plano, el personal docente que desarrolla clases en contextos vulnerables debe generar prácticas pedagógicas focalizadas en estrategias de aprendizaje, enmarcadas en las características y necesidades del alumnado que está en situación de vulnerabilidad, a fin de desarrollar su capacidad reflexiva y de autonomía en la generación de aprendizajes. Un tercer profesor, en el desarrollo de la enseñanza expone el tema combinándolo con el uso de esquemas e imágenes, sus estudiantes logran tomar apuntes ya que el ritmo de la clase así lo permite, el ambiente de luz es el adecuado. Durante todo el desarrollo el docente realiza solo dos preguntas a la audiencia, las cuales el mismo docente va respondiendo, y redondeando la respuesta, como se presenta en los escritos etnográficos: “... ¿Qué más?, ¿es un recurso natural?..¿A qué tipo de recurso natural pertenece la madera?, el estudiante se queda callado...luego el docente responde 
las preguntas...” (OB/8: P1). En tal sentido, la participación activa de estudiantes en el proceso de enseñanza y aprendizaje no se genera en el modelo de desarrollo de la clase, dado que no existe intervención espontánea de estudiantes durante la clase y, en el caso de la realización de las preguntas, estas son de respuesta corta y son explicadas por el propio docente, sin dejar espacio para la reflexión estudiantil. La finalización de la clase ocurre de forma inmediata sin la aplicación de criterios evaluativos de efectividad de la clase, y sin una etapa de conclusiones y de reflexión. Al respecto, Shulman (2005) plantea que la docencia se inicia cuando se "reflexiona", en qué es lo que debe ser aprendido y cómo debe ser aprendido por el estudiantado. En consecuencia, se aprecia una falta de variedad en el uso de estrategias y un manejo inadecuado de estas, lo que evidencia debilidades en el manejo del conocimiento didáctico contenido (CDC). Por lo tanto, desde el contexto vulnerable, el personal docente debe poseer un dominio didáctico del contenido, es decir, debe portar un conocimiento propio de cómo enseñar una materia en contextos vulnerables. Desde esta perspectiva, el profesorado debe emplear una diversidad de estrategias y técnicas didácticas, actividades y recursos didácticos, asimismo e innovar en este campo constantemente, para contribuir a que el estudiantado desarrolle el pensamiento reflexivo, crítico y divergente, además la capacidad de autonomía, y el aspecto emocional con la finalidad de que logren anclar los aprendizajes.

\section{Categoría 3. Dominio del contenido disciplinario}

En esta categoría, se visualiza un débil manejo por parte de docentes de los contenidos disciplinarios y del conocimiento didáctico del contenido. Al respecto, se visualiza un uso reiterado de texto escolar, donde con cierta frecuencia dictan la materia de este recurso didáctico y del mismo modo ocurre con las presentaciones (power point); focalizan el desarrollo de prácticas de enseñanza en lo vertical y en la reproducción del conocimiento, enmarcándose en un enfoque conductista y, como consecuencia, se aprecia un escaso dominio del contenido disciplinario: “...Yo, uso Power Point, que es una herramienta nueva y bastante fácil de manejar, y más cómoda para enseñar los contenidos" (E: P15). Por otra parte, un segundo docente declara, "yo utilizo bastante el texto, generalmente a uno le falta tiempo para hacer guías o actividades más didácticas (E: P14). Otro profesor expresa: "saquen el texto, y lean la lectura de la página 17 y realicen las actividades que están a continuación” (OB/10: P6). En razón de las evidencias, es posible señalar que el personal docente no tiene un manejo adecuado del conocimiento disciplinar del contenido, para llevarlo cabo a la enseñanza durante el desarrollo de la clase. En concordancia con lo expuesto, se aprecia la falta de perfeccionamiento que tiene en las disciplinas en las que enseña. Esto queda reflejado en la siguiente evidencia empírica: “... nosotros tenemos como profesores, un papel mucho más relevante en este proceso de enseñar y captar conocimiento en los alumnos ... entonces tenemos que tener herramientas para poder llegar a ellos, tenemos que conocer sus tipos de aprendizajes, de manera que debemos capacitarnos constantemente" (E: P2).

En general se observa a docentes con un débil manejo en el contenido disciplinario y en la trasposición didáctica. Del mismo modo, se visualiza falta de perfeccionamiento en las disciplinas que enseñan. En este contexto, Shulman (2005) sostiene que el profesorado debe manejar adecuadamente el conocimiento del contenido. Es decir, portar un dominio del conocimiento erudito de una materia, para enseñar adecuadamente en contextos vulnerables. Al respecto, Gonzaga (citado en Mansilla y Beltrán, 2013), sostiene que se requiere que posea un conocimiento amplio y profundo de cómo y cuándo enseñar, con un manejo apropiado de estrategias de enseñanza y aprendizaje, de procedimientos e instrumentos de evaluación. En 
tal sentido, el profesorado debe convertirse en un maestro o maestra en la enseñanza de una disciplina, para entregar adecuadamente los saberes y generar aprendizajes de calidad, además solucionar problemas, y mejorar la realidad formativa y educativa de sus estudiantes en situación de vulnerabilidad.

\section{Categoría 4. Concepción de evaluación}

La mayoría de docentes que se entrevistaron señala que la evaluación está asociada a la medición y calificación. Ello se observa en las siguientes textualidades: "Evaluar es medir la recogida de información de los aprendizajes por parte de los alumnos" (E: P1). "Es la valoración del procesos de enseñanza aprendizaje para luego convertirse en calificación” (E: P16).

En el discurso docente no aparece el concepto de evaluación como un todo, sino fragmentado en los términos de "recogida de información" para luego "medir" y "calificar" los aprendizajes, en tal sentido la evaluación se reduce a actos técnicos y al control. En contraste con lo anterior, Castillo y Cabrerizo (2003) plantean que la evaluación es un punto de encuentro del acto didáctico o del proceso de enseñanza, debido a que el personal docente comunica los objetivos a lograr con sus estudiantes; el encuentro didáctico requiere el diálogo, la intercomunicación y la interacción docente-estudiante, donde la base de este punto de encuentro es la evaluación, pues guía la enseñanza en función de los datos que manifieste; permite revisar prácticas y conocer características del alumnado y sus ritmos; analiza los problemas que surgen en la explicación y adquisición de conocimientos; y valora sus actitudes, capacidades y habilidades. Para Ahumada (2001), la evaluación debe ser un proceso continuo, dinámico, flexible, reflexivo e inherente a todo aprendizaje. Desde esta lógica, el personal docente debe tener un dominio disciplinar de la evaluación, la cual debe estar enmarcada en un carácter, continuo, dinámico, holístico y, además, debe centrarse en los aprendizajes, de manera que responda a las necesidades educativas y características de estudiantes en condiciones de vulnerabilidad.

\section{Categoría 5. Prácticas evaluativas}

En esta categoría, se evidencia que para la mayoría del profesorado la prueba diagnóstica no es importante para el diseño y orientación de las clases, lo cual se relaciona con el modelo conductista. Lo anterior se refleja en la siguiente textualidad: "La evaluación diagnostica no muestra los conocimientos que debería tener el alumno, en general los resultados son bajos...” (E: P19). Tal evidencia denota un desconocimiento teórico, y práctico por la parte docente, respecto de este tipo de evaluación, lo cual resulta preocupante, puesto que este tipo de evaluación es relevante para diagnosticar los aprendizajes de estudiantes que presentan dificultades en el rendimiento y el desempeño educativo, esto con la finalidad de tener información para entregar una enseñanza adecuada.

Por otra parte, se visualiza una débil aplicación de evaluaciones centrada en los procesos pedagógicos que permitan obtener información para la mejora de la calidad de los aprendizajes. Por lo tanto, la práctica evaluativa se centra en los resultados, manifestándose en el siguiente relato: "la evaluación se aplica con mayor frecuencia al final de las actividades realizadas para ver los resultados" (E: P9). El proceso evaluativo no está centrado en la continuidad y permanencia del proceso, sino que se emplea con mayor presencia al final del proceso educativo. Ahumada (2001) señala que la evaluación debe estar centrada en la continuidad y permanencia, más que en un momento, y que debe tener un carácter retroalimentador. Entonces, la evaluación debe estar ajustada al proceso de aprendizaje desde una perspectiva integral y no enmarcada en un proceso deshumanizador centrado en los resultados y en el control.

En suma, las practicas evaluación empleadas en estudiantes en condiciones de vulnerabilidad deben convertirse en una herramienta capaz de diagnosticar y movilizar aprendizajes de calidad; desde una lógica, 
dinámica, flexible, cíclica; sobre la base teórica, práctica, reflexiva y crítica con un perfil retroalimentador y dialógico entre docente y estudiante. Asimismo, debe centrase en las capacidades, estilos de aprendizajes, valores, afectividad, y en las emociones del estudiantado.

\section{Categoría 6. Liderazgo pedagógico}

Esta categoría evidencia una ausencia de liderazgo en el profesorado: "Nosotros los profesores a menudo tenemos que solucionar diversos problemas, pero a veces no tenemos el liderazgo para dar respuestas a ciertas acciones o problemas que suceden en el aula..." (E: P15). En este contexto, surge la evidencia de que la mayoría declara no poseer algún tipo de liderazgo. Esto queda reflejado en la siguiente narración: "Creo a nosotros nos falta poseer algún tipo de liderazgo para enfrentar nuestras clases" (E: P5). "La mayoría de nosotros no manejamos ningún tipo de liderazgo, por lo que nos deberían capacitar en este tipo de materias...” (El: P8).

Respecto a las evidencias, Leithwood (2009, p. 20) expresa que el liderazgo se enfoca en "la labor de movilizar e influenciar a otros para articular y lograr las intenciones y metas compartidas de la escuela". En este sentido, la (OCDE, 2008) señala que líderes con orientación en lo pedagógico tienen su impacto real y efectivo tanto en el cuerpo de docentes como en los alumnos y alumnas de los centros educativos. La revisión académica identifica el liderazgo de organizaciones educativas como una de las principales variables que inciden en un mejor desempeño de los establecimientos y del sistema en general. En este plano, Anderson (2010) plantea que el liderazgo pedagógico, a nivel de escuelas desempeña un rol altamente significativo en el desarrollo de cambios en las prácticas docentes, en la calidad de estas, y en el impacto que presentan sobre la calidad del aprendizaje del alumnado. Al respecto, desde un contexto vulnerable, el liderazgo pedagógico permite influir positivamente en estudiantes durante el proceso de enseñanza y aprendizaje, debido a que fortalece la confianza, autonomía y la capacidad de trabajo en equipo entre docentes, docenteestudiante y entre estudiantes. Asimismo, contribuye al fortalecimiento ético y valórico y al desarrollo del pensamiento reflexivo, crítico y divergente en las diversas actividades en las cuales participa el estudiantado. En este contexto, Robinson, Hohepa, y Lloyd (2009) manifiestan que los efectos de determinados modos de ejercicio del liderazgo son aún mayores en escuelas situadas en contextos vulnerables y de pobreza, donde un buen liderazgo educativo puede contribuir decididamente a incrementar sus índices de mejora. En suma, el liderazgo pedagógico es una capacidad esencial que debe estar presente en las prácticas educativas del personal directivo y docente, y en las actividades que desarrolla el estudiantado, con el fin de que contribuir a mejorar los aprendizajes y al desarrollo de la formación del alumnado desde un enfoque integral.

\section{Conclusión}

A partir de la discusión de los resultados, es posible dar respuesta al objetivo propuesto para este trabajo. Al respecto, se constata que dentro del proceso de "diseño de la planificación de enseñanza", los modelos observados son más bien rígidos y con una lógica predeterminada, puesto que el personal docente no utiliza instancias para realizar adecuaciones durante su aplicación orientadas a la práctica reflexiva, de modo que el alumnado ocupa un rol más bien pasivo. Asimismo, se observa un desconocimiento del contexto y de las habilidades, capacidades, estilos y ritmos de aprendizajes de estudiantes a la hora de planificar. Respecto a esto, De Miguel-Díaz (2005) señala que la enseñanza debe estar centrada en el alumnado y en sus capacidades, en tal sentido, la planificación debiera sufrir un cambio en esta dirección.

Otro punto obstaculizador que se observa es la ausencia de trabajo colaborativo y reflexión pedagógica entre docentes, gatillado por la falta de condiciones de organización óptimas por parte del personal directivo. En suma, se puede concluir que no se observan diseños de planificación orientados en torno al estudiantado que está en situación de vulnerabilidad educativa, dado que no se visualiza un trabajo articulado, colaborativo 
ni reflexivo entre el profesorado, debido a la falta de condiciones de organización óptimas, lo que dificulta el proceso de enseñanza y aprendizaje. En tal sentido, el diseño de la planificación no debe dejar espacios para la improvisación y las creencias.

Respecto a las "estrategias didácticas" que emplea el profesorado, estas obstaculizan la generación de aprendizajes en estudiantes, dado que se focalizan mayoritariamente en el enfoque tradicionalista, centradas en clases expositivas, las cuales carecen de una estructura didáctica enmarcada en paradigmas actuales de enseñanza y aprendizaje. Esto, porque el profesorado tiene figura de autoridad, posee el conocimiento y la verdad absoluta y, el estudiantado se considera un actor pasivo. En tal condición, no existen espacios para la reflexión, diálogo y de retroalimentación de los aprendizajes durante el desarrollo de la clase; estas se alejan del modelo de transposición didáctica contextualizada y se acerca más a un modelo de transferencia de información de forma mecánica, puesto que no considera el amplio bagaje de estrategias didácticas centradas en el aprendizaje. Asimismo, se aprecia un manejo inadecuado de algunas de estas, evidenciándose debilidades en el dominio del conocimiento didáctico contenido (CDC). Para revertir lo expuesto, de acuerdo con Bolívar (2005), el profesorado debe desarrollar un conocimiento específico: cómo enseñar su materia específica, por lo que se requiere un CDC, propio del buen hacer docente. En este plano, el personal docente debe generar prácticas pedagógicas innovadoras focalizadas en estrategias de aprendizaje, y recursos didácticos centrados en potenciar el desarrollo del pensamiento reflexivo, crítico y divergente y la capacidad de autonomía. Además, debe atender las características y necesidades de sus estudiantes en condiciones de vulnerabilidad, con la finalidad de que estos grupos se apropien de los aprendizajes.

En la categoría "dominio del contenido disciplinario" se evidencia debilidad del profesorado en el dominio del conocimiento del contenido (CC) en las disciplinas que enseña, donde se vale del uso excesivo del texto y proyecciones (Power Point) y, con cierta frecuencia, dicta la materia de estos recursos didácticos. Además, se constata su falta de perfeccionamiento en las disciplinas que enseña. El personal docente, al no tener un dominio del conocimiento erudito de una materia, dificulta los procesos de enseñanza y aprendizaje y, por tanto entorpece el rendimiento y el desempeño educativo, la formación y educación de estudiantes que presentan diversas carencias en el aspecto social y cultural.

Respecto de la "concepción de evaluación", en el discurso docente no aparece el concepto de evaluación como un todo, sino fragmentado en los términos de "recogida de información" para luego "medir y calificar los aprendizajes". Entonces, el razonamiento de la acción evaluativa se reduce a actos técnicos y al control. Esto es ocasionado por un desconocimiento disciplinar y débil manejo de los tipos de evaluación, sumado a lo cual se visualiza la falta de vinculación de la evaluación respecto de la consideración de las necesidades educativas y características de estudiantes en condiciones de vulnerabilidad. En tal sentido, se concluye que la concepción de la evaluación que posee el profesorado es fragmentada y descontextualizada, por tanto, desfavorece, de forma directa, los aprendizajes estudiantiles.

En cuanto a la "práctica evaluativa", se puede concluir que se focaliza en los resultados y en el control, y no en la continuidad y permanencia del proceso de enseñanza y aprendizaje; además, se presenta como un proceso deshumanizador. En tal sentido, la evaluación se realiza como una necesidad docente, y no como un proceso continuo, retroalimentador, programado, dinámico, dialógico, centrado en las capacidades, estilos de aprendizajes, valores, y en la afectividad del alumnado. En consecuencia, la práctica evaluativa dificulta la formación de estudiantes que presentan dificultades en el rendimiento y el desempeño educativo desde un aspecto, teórico, practico, reflexivo, crítico, valórico, social, cultural y afectivo.

Finalmente, respecto del "liderazgo pedagógico", la mayoría de docentes declara no poseer algún tipo de liderazgo que le permita solucionar problemas y llevar a cabo eficazmente acciones pedagógicas encaminadas al logro de aprendizajes de calidad en el aula. Además, la ausencia de la práctica de un liderazgo pedagógico en contextos vulnerables no favorece al desarrollo de la confianza, reflexión crítica y la capacidad de trabajo en equipo entre profesorado, docente-estudiante y entre estudiantes. Por lo tanto, dificulta una formación adecuada de estudiantes desde un plano integral. 
Las conclusiones desde las evidencias empíricas resultan preocupantes, si se piensa que profesores y profesoras son los actores principales del proceso de enseñanza y aprendizaje de sus estudiantes. Por lo tanto, docentes que se desempeñan en contextos vulnerables deben poseer un conocimiento disciplinar, didáctico y del contexto, a fin de movilizar procesos de transposición didáctica eficaces. Entonces, surge el desafío de la superación de una enseñanza centrada en el profesorado, de tal forma que esta se oriente a estudiantes. Para ello, el personal docente debe ser capaz de emplear diseños instruccionales, flexibles y dialógicos donde se considere al estudiantado un actor principal en la construcción de su propio aprendizaje.

En consecuencia, es apremiante centrar y profundizar las investigaciones en torno a los procesos de trasposición didáctica que desarrollan docentes en escuelas rurales en condiciones vulnerables, con el fin de tener evidencias que permitan la toma de medidas orientadas a la mejora del proceso enseñanza y aprendizaje.

Finalmente, este estudio proyecta contribuir a la reflexión crítica en relación con la profesionalización docente respecto del desarrollo de sus procesos de transposición didáctica en contextos de vulnerabilidad, para garantizar una formación y educación integral y, además, evitar dificultades e interrupciones en la trayectoria escolar, y disminuir las desigualdades sociales y educativas de estudiantes en condiciones de vulnerabilidad. En este sentido, el personal docente debe ser capaz de hacerse cargo de una pedagogía contextualizada y social, a fin de provocar transformaciones sociales en contextos educativos vulnerables.

\section{REFERENCIAS}

Ahumada, P. (2001). La evaluación una concepción de aprendizaje significativo. Valparaíso: Salesianos.

Acevedo, J. (2009). Conocimiento didáctico del contenido para la enseñanza de la naturaleza de la ciencia (I): El marco teórico. Revista Eureka sobre Enseñanza Divulgación de las Ciencias, 6(1), 21-46. Recuperado de http:// www.redalyc.org/pdf/920/92012998003.pdf

Anderson, S. (2010). Liderazgo directivo: Claves para una mejor escuela. Revista Psicoperspectivas, 9(2), 34-52. Recuperado de http://www.scielo.cl/pdf/psicop/v9n2/art03.pdf

Aroca, M. (2015). Causas que afectan a grupos de vulnerabilidad educativa: Soluciones desde la pedagogía sociocrítica. Provincia Bolívar. Revista Ciencias Pedagógicas e Innovación, 3(3), 7-12.

Aylwin, M., Muñoz, A., Flanagan, A. y Ermter, K. (2005). Buenas prácticas para una pedagogía efectiva. Santiago, Chile: UNICEF.

Becerra, S., Mansilla, S. Saavedra, J., Tapia, C. (2012). Liderazgo de los directivos docentes en contextos vulnerables. Revista Educación y Educadores, 14 (2), 389-409.

Bisquerra, R. (2004). Metodología de la investigación educativa. Madrid: La Muralla.

Bolívar, A. (2005). Conocimiento didáctico del contenido y didácticas específicas. Profesorado. Revista de currículum y formación del profesorado, 9(2), 1-39. Recuperado de https://www.ugr.es/ recfpro/rev92ART6.pdf

Böhm, W.y Schiefelbein, E. (2010). Repensar la educación. Diez preguntas para mejorar la docencia (2a ed.). Santiago, Chile: Salesianos Impresores.

Camilloni, A. Cols, E., Besabe, L. y Feeney, S. (2007). El saber didáctico. Buenos Aires: Paidós.

Castillo, S. y Cabrerizo, J. (2003). Evaluación educativa y promoción escolar. Madrid: Pearson.

Castro, F. Domínguez, C. Manzano, M., Zavala, P. Sánchez, M. y Guzmán, B. (2015). Causas que afectan a grupos de vulnerabilidad educativa: soluciones desde la pedagogía sociocrítica. Provincia Bolívar. Revista Ciencias Pedagógicas e Innovación, 3(3), 7 - 12. Recuperado de http://www.upse.edu.ec/rcpi/index.php/revistaupse/ article/view/69

Cerda, H. (1998). Los elementos de la investigación. Bogotá: El Búho limitada.

Chevallard, Y. (1991). La transposición didáctica. Del saber sabio al saber enseñado. Buenos Aires: Aiqué.

Clermont, C., Borko, H. y Krajcik, S. (1994). Comparative study of the pedagogical content knowledge of experienced and novice chemical demonstrators. Journal of Research in Science Teaching, 31(4), 419-441. 
Cochran-Smith, M. \& Fries, K. (2005). The AERA Panel on Research and Teacher Education: Context and Goals. En M. Cochran-Smith \& K. Zeichner (Eds.), Studying Teacher Education. The Report of the AERA Panel on Research and Teacher Education [El estudio de la formación del profesorado. El Informe del Panel de AERA de Investigación y Profesor de Educación], (pp. 37-68). New Jersey: Lawrence Erlbaum Associates.

Denzin, N. (1970). Sociological methods. [Métodos sociológicos], Chicago: Aldine

Denzin, N. (1989). The Research Act. (3a ed.). [La Ley de Investigación]. Englewood Cliffs, NJ: Prentice Hall

De Miguel-Díaz, M. (2005). Cambio de paradigma metodológico en la educación superior. Exigencias que conlleva. Cuadernos de Integración Europea, 2, 16-27.

De la Torre, F. (2005). Doce lecciones de pedagogía, educación y didáctica. México: Alfaomega.

Dirección General de Cultura y Educación. (2009). Definiciones para la comprensión de situaciones de vulnerabilidad educativa. Dirección Provincial de Planeamiento, Provincia de Buenos Aires, Argentina. Recuperado de http:// www.region11.edu. ar/publico/portal/doc/biblioteca/vulnerabilidad-educativa.pdf

Dirección General de Cultura y Educación. (2010). Definiciones de vulnerabilidad. Dirección Provincial de Planeamiento, Provincia de Buenos Aires, Argentina. Recuperado de http://servicios2.abc.gov.ar/lainstitucion/ organismos/direccionprovincialplaneamiento/destacadoserie/definiciones-de-vulnerabilidad.pdf

Flick, U. (2007). Introducción a la investigación cualitativa. Madrid: Morata.

Fullan, M. (2003). Change forces with a vengeance. Londres: Falmer Press.

Gallego, J. y Salvador, F. (2012). El diseño didáctico: Objetivos y fines. En A. Medina y F. Salvador, Didáctica general. Madrid: Pearson Educación.

Gess-Newsome, J. (1999). Pedagogical Content Knowledge: An introduction and orientation. En Julie GessNewsome y Norman Lederman (Eds.), Examinig Pedagogical Content Knowledge, [Examinando el conocimiento didáctico del contenido], Dordrecht: Kluwer Academic Publishers.

Giberti, E., Garaventa, J. y Lamberti, S. (2005). Vulnerabilidad, desvalimiento y maltrato infantil en las organizaciones familiares. Buenos Aires: Centro de Publicaciones Educativas y Material Didáctico.

Gurdián-Fernández, A. (2007). El paradigma cualitativo en la investigación socioeducativa. San José, Costa Rica: Print Center.

Grizales-Franco, L. y González-Aguadelo, E. (2009). El saber sabio y el saber enseñado: Un problema para la didáctica universitaria. Revista Educación y Educadores, 12(2), 77-86.

Kuhn, T. (1962). La estructura de las revoluciones científicas. D. F., México: Fondo de Cultura Económica.

Leithwood, K. (2009). ¿Cómo liderar nuestras escuelas? Aportes desde la investigación. Santiago, Chile: Impreso en Salesianos.

León, F. (2011). Pobreza, vulnerabilidad y calidad de vida en América Latina. Retos para la bioética. Acta Bioethica, $17(1), 19-29$.

Marcelo, C. (2001). El aprendizaje de los formadores en tiempos de cambio. La aportación de las redes y el caso de la Red Andaluza de Profesionales de la Formación. Profesorado. Revista de Currículum y Formación de Profesorado, 5(1), 1-17.

Mansilla, S. y Beltrán, J. (2013). Coherencia entre las estrategias didácticas y las creencias curriculares de los docentes de segundo ciclo, a partir de las actividades didácticas. Perfiles Educativos, 35(139), 25-39. Recuperado de http:// www.redalyc.org/articulo.oa?id=13225611012

Marhuenda, F. (2000). Didáctica general. Madrid: De la Torre.

Mayorga, A., Enrique, S. y Rodríguez, J. (2014). Comparación técnica entre Atlas. Ti 7 y N-Vivo 10: Software para el análisis de datos cualitativo. Universidad Nacional de Colombia. Bogotá, Colombia.

Morgado, C. (2007). El arte de saber explicar y enseñar. Recuperado de http://angepituf871.blogspot.com/2007/07/ el-arte-de-saber-explicar-y-ensear.html

MIDEPLAN. (2002). Síntesis de los principales enfoques, métodos y estrategias para la superación de la pobreza. Santiago. Ministerio de Planificación y Cooperación. 
Moreno, T. (2011). Didáctica de la educación superior: Nuevos desafíos en el siglo XXI. Perspectiva Educacional, Formación de Profesores, 50(2), 26-54.

Moreno, J. Rodríguez, G., Mera, E., y Beltrán, L. (2007). Estrategias didácticas desarrolladas por los docentes para orientar el trabajo integrador en la facultad de Psicología. Revista de psychología, 1, 133-162.

Mulholland, J. y Wallace, J. (2005). Growing the tree of teacher knowledge: Ten years of learning to teach elementary science, Journal of Research in Science Teaching, 42(7), 767-790.

Murillo, J. (2005). La investigación en eficacia escolar y mejora de la escuela como motor para el incremento de la calidad educativa en Iberoamérica, Revista Electrónica Iberoamericana sobre Calidad, Eficacia y Cambio en Educación, 3(2), 1-12. Recuperado de http://www.uam.es/personal_pdi/stmaria/jmurillo/recursos/Efi cacia_en_Ib.htm

OCDE. (2004). Teachers matter: attracting, developing and retaining effective teachers. [La cuestión del profesorado: Atraer, desarrollar y retener a los maestros eficaces], Paris: OCDE.

OCDE. (2008). Education and training policy. Improving school leadership. [La educación y la política de formación. Mejorar el liderazgo escolar], Policy and Practice. Paris: OCDE.

Pellón, M., Mansilla J. y San Martín, D. (2009). Desafíos para la transposición didáctica y conocimiento didáctico del contenido en docentes de anatomía: Obstáculos y Proyecciones. Revista International Journal of Morphology, 27(3) 743-750.

Raczynski, D. y Muñoz, G. (2005). Efectividad y cambio escolar, en condiciones de pobreza en Chile, Santiago: Gobierno de Chile-Ministerio de Educación.

Robinson, V., Hohepa, M. y Lloyd, C. (2009). School leadership and student outcomes: Identifying what works and why: Best evidence synthesis iteration (BES). New Zealand: Ministry of Education.

Ruiz, J. (2003). Metodología de la investigación cualitativa. (3ª ed.) España: Universidad de Deusto.

Sabuda, F. (2009). ¿Quién es vulnerable en la escuela? Análisis territorial de rendimientos educativos y contexto sociocultural en el Partido de General Pueyrredón, Argentina. Revista Colombiana de Geografía, 18, 45-57. Recuperado de file://C:/Users/Juan\%20Beltran/Downloads/

Sandín, M. (2003). Investigación cualitativa en educación. Fundamentos y tradiciones. Madrid: Mc Graw Hill.

Schön, D. (2011). Una práctica profesional reflexiva en la universidad. Revista Compás Empresarial, 3(5), 54-58. Recuperado de http://qacontent.edomex.gob. $\mathrm{mx} /$ idc/groups/public/documents/edomex_archivo/ dregional_tol_pdf_docfor_09.pdf

Shulman, L. S. (1986). Those who understand: knowledge growth in teaching. Educational Researcher, 15(2), 4-14. Traducción castellana (2005): El saber y entender de la profesión docente. Estudios Públicos, 99, 195-224.

Shulman, L. (2005). Conocimiento y enseñanza: Fundamento de la nueva reforma. Profesorado. Revista de Currículum y Formación de Profesorado, 9(2), 1-30. Recuperado de https://www.ugr.es/ recfpro/ rev92ART1.pdf

Strauss, A. y Corbin, J. (2002). Bases de la investigación cualitativa. Técnicas y procedimientos para desarrollar la teoría fundamentada. Medellín, Colombia: Universidad de Antioquia.

Tardy, M. (1995). La transposition didactique. En J. Houssaye (ed.), La pédagogie: une encyclopédie pour aujourd'hui. [La pedagogía: una enciclopedia para hoy] Paris: ESF.

UNESCO. (2010). Factores asociados al logro cognitivo de los estudiantes de América Latina y el Caribe. Organización de las Naciones Unidas para la Educación la Ciencia y la Cultura. Santiago, Chile. Recuperado de http://unesdoc.unesco.org/images/0019/001902/190213s.pdf

Valles, M. (2007). Técnicas cualitativas de investigación social. Reflexión metodológica y práctica profesional. Madrid: Síntesis.

Verret, M. (1975). Le temps des études. Paris: Librairie Honoré Champion.

Wisner, B., Blaikie, P., Cannon, T. y Davis, I. (2004). At risk: natural hazards, people's vulnerability and disasters, Londres: Routledge. 
Juan Beltrán Véliz, et al. Prácticas que obstaculizan los procesos de transposición didáctica en e...

Zambrano, L. (2006). Tres tipos de saber del profesor y competencias: Una relación compleja. Revista Educere, 10(33), $225-232$.

CC BY-NC-ND 